\title{
Research on Emotional Labor and Management of Takeaway Delivery Staff
}

\author{
Fulin Xiang* and Wenbing $\mathrm{Wu}$ \\ School of Economics and Management, Beijing Jiaotong University, Beijing, China \\ ${ }^{*}$ Corresponding author. Email: 20120710@bjtu.edu.cn
}

\begin{abstract}
Takeaway delivery staff have made a huge contribution to the last mile of service in China's catering industry. At the end of 2019, the number of delivery staff officially registered on the takeaway platform has reached 7 million. Most of the existing research is the identification and labor process of takeaway delivery staff. To discuss and divide, the emotional labor and management research carried out by the delivery staff as the research object is still blank. As the main body of the takeaway service provider, this group of employees has heavy emotional labor problems. Emotional labor not only affects employees' physical and mental health and work performance, but also affects organizational goals and normal operations. This article starts from the perspective of emotional labor, combines existing literature, and divides the emotional labor content of delivery workers from the perspective of organization, business, and social rules, and discusses the management and optimization of emotional labor from the organizational and employee levels.
\end{abstract}

Keywords: Emotional labor, Takeaway delivery staff, Performance management, Psychological capital.

\section{INTRODUCTION}

Since the combination of "Internet + " and the traditional catering industry, the food and beverage industry has been developing rapidly in just a few years, and the food and beverage industry has become an important driving force for China's consumption growth. In 2019, the size of China's catering and takeaway industry reached 653.6 billion yuan, up 39.3\% from 2018. There are about 460 million takeout consumers in China. In February 2020, "online delivery person" officially became a new occupation and was included in China's national occupational Classification catalog. Millions of the new generation of workers, often referred to as "takeout boys", have identified their jobs. Takeaway delivery staff who sell outside as delivery objects have made great contributions to China's social and economic development and the last mile service of the catering industry. Comb in recent years, however, takeaway delivery staff related news reports, in addition to the mainstream media for their contribution to the work of singing, reflecting the takeaway delivery staff with customers, merchants, security and other conflicts because of emotional problems, article also abound in the emotional labor and delivery and distribution staff management should pay more attention to and attention.

\subsection{Research Background}

A takeout deliverer belongs to an online deliverer. According to the definition of occupation, they are service personnel engaged in receiving and checking customer orders through mobile Internet platforms, etc., and delivering the orders to the designated place within a certain period of time according to the order requirements and according to the intelligent planning route of the platform. In the work characteristics, has a strong "meal effect", localization, life circle color. By the end of 2019, there are 7 million employees engaged in delivery work in China, among which 3.987 million are employed under Meituan platform and 3 million are registered under ELEME hummingbird distribution platform. According to the survey, $75 \%$ of takeaway delivery staff are from rural areas, and $82 \%$ are born in the 1980s and 1990s, showing the characteristics of "young migrant workers".

At present, China's takeaway deliverers are mainly dedicated deliverymen and crowdsourcing deliverymen. Dedicated deliverymen are system deliverymen, and 
there is no need for deliverymen to scramble for orders. Crowd-sourcing deliverers can register to receive orders on multiple delivery platforms to increase their income. The delivery process of a takeaway deliveryman can be roughly divided into three steps: receiving orders, receiving orders and delivering orders. Just from the perspective of the working process, the whole delivery work is not complicated and can be completed step by step.

\subsection{Literature Review}

At present, academically, the identity identification and labor process of delivery personnel for external sales have been discussed and divided. It has been found that delivery personnel can be divided into three types: direct delivery personnel, crowdsourcing delivery personnel, and special delivery personnel. The working process can be roughly divided into three steps: receiving orders, picking up meals at the store, and delivering orders. The labor of a takeaway deliverer is a comprehensive compound labor, which requires not only physical labor and mental labor, but also emotional labor and emotional labor [1]

Emotional labor is regarded as the third kind of labor besides physical labor and mental labor. Emotional labor by sociologists Hochschild put forward in 1983, For reward, employees manage their own emotions, and in accordance with the requirements of the organization of facial expressions or body language to show, in order to provide a good "customer service" and produce false behavior to hide or suppress negative personal feelings, to eventually acquire value in exchange for the purpose [2]. In other words, emotional labor is sold for wages and has exchange value. Hochschild proposes two strategies of surface behavior and deep behavior of emotional labor based on the psychological process of emotional labor. Surface behavior refers to the emotion that an individual expresses externally and does not experience subjectively. Deep behavior refers to an individual's ability to change his or her inner feelings in order to experience the emotions needed by the organization. Hochschild also proposes another emotional labor strategy, in which the employees' real emotional feelings are in line with the emotional expression rules of the organization. It is called passive deep behavior, and he thinks that this strategy does not require any effort. In contrast to passive deep behavior, deep behavior is described as active deep behavior[3]

Empirical studies have confirmed that emotional labor not only affects employees' physical and mental health and work performance, but also affects the achievement of organizational goals and even the survival and development of organizations [4]. However, the research on emotional labor and management carried out by delivery personnel for foreign sales is still blank.

\section{FORMATION OF EMOTIONAL LABOR FROM THE PERSPECTIVE OF RULES}

Delivery platform through algorithm to spy on takeaway delivery staff physical labor, under the application of mobile Internet technology, takeaway delivery staff orders, pickup, delivery, etc. Series of action became a string can be quantified in the flow state of spatio-temporal data, can be comprehensive supervision and management, its inner invisible emotional labor and mental labor but less people can pay attention to. As a matter of fact, the emotional labor of a delivery worker is equal to the physical labor of a delivery worker when facing different people.

\subsection{Emotional Labor under the Algorithm Rules}

In order to attract, grab and retain customers, selling platform at home and abroad all the time as a lifeline of food distribution services, the platform the labels "realtime, high efficiency", "half an hour the whole city", "average 28 minutes" to attract customers eye, also constantly to takeaway delivery staff exerting stress distribution of work. Many takeaway delivery staff said platform and site requirements and shorter delivery time, room $3 \mathrm{~km}$ distance, for example, Meituan takeaway meals in 2016 is the longest time limit for 1 hour, 45 minutes into the 2017, 2018, and shortened the 7 minutes, at 38 minutes, in 2019, the longest delivery time is pressed up to 30 minutes [5]. Meituan real-time intelligent delivery system is called super brain, ELEME is named ark. For the creator, it is the progress of AI intelligent algorithm deep learning, while for the delivery staff, it is the source of their emotional stress. Unreasonable delivery route navigation is added new risks for takeaway delivery member and emotional stress, algorithm to predict the length of time is based on the linear distance point-to-point, real buildings around with traffic lights, traffic line and has not been taken into account, sometimes, only distribution navigation actively guide the takeaway delivery staff retrograde, through the electric car driving bridge is prohibited. This leaves delivery workers with a dilemma in the delivery process, whether to obey traffic rules but be late for delivery, pay a fine or take a fluke to complete the delivery quickly. The expected delivery time of the customer side is inconsistent with the delivery time required by the deliveryman side, which lays the foundation for the contradiction and conflict between the deliveryman and the customer. The time standard and delivery rules formulated by the platform accelerate the formation of emotional labor of deliverers. 


\subsection{Emotional Labor under the Algorithm Rules}

Under the background of the rise of platform capital, the difference between normalization and standardization of commercial services is getting smaller and smaller. In the takeaway delivery industry, driven by the requirements and interests of commercial rules, takeaway deliverymen need to make every emotional effort to improve customers' perception of service quality in order to expect favorable comments from customers[6]. The first thing to say on the phone is 'Hello, thank you for ordering takeout, I'm your deliveryman', the second thing to say on delivery is 'This is your takeout, sorry to keep you waiting', or 'Thank you for ordering $\times \times$ takeout, have a nice meal,' smile on your face and put your food in your hands... Call ahead and wait for a response if no one is answering. Customers and takeaway delivery staff is still in the permissions on the unequal status, customers can have can view all distribution member name, contact address, instant permissions, can call urged, cancel the order, or complaint distribution, and some customers will also put forward many additional accessory requirements, such as a bottle of water, draw a pig page, or you give bad review, takeaway delivery staff in dealing with these situations require more emotional labor, let customer satisfaction, to be bad review or get high praise, this is an integral part of their emotional labor.

Merchants entering the delivery platform, in some cases, will also affect the emotional labor of the delivery staff. If a merchant has a large number of orders, the delivery speed is slow, or the food is delivered out of order, the deliveryman who has received the order can only appease or comply with the merchant's behavior under the commercial rules by smiling face or selfconfessed unlucky emotional labor, which is also a part of the emotional labor of the delivery clerk.

\subsection{Emotional Labor under Social Rules}

Deliverymen also need to follow social rules for emotional labor. The traffic police, security guards and community owners under social rules are also the objects that deliverymen often deal with. Many deliverymen go the wrong way because they are in a hurry to deliver food and are in a hurry. After being stopped by the traffic police, they need to speak up, admit their mistakes and plead for mercy in order to reduce or avoid punishment. Similarly, takeout deliverymen need to ingratiate themselves with security guards, exchanging friendly words, snagging and offering cigarettes in exchange for access to companies, neighborhoods and schools. In the face of community orders such as temperature taking and registration set by owners and managers of various communities, deliverymen working against time still have to appease them with flexible and changeable smiling faces. Even so, no amount of emotional labor can change social rules, such as restricting deliverymen's use of passenger elevators or banning them from riding electric cars into the community, when they are encroachment on too much public resources. What's more, the labor situation of delivery workers is constantly changing, which means that new orders and new emotional labor will be born, and the old emotional labor may no longer play a role, and emotional labor is in the change and change.

As a member of the society, takeout deliverymen themselves are mostly shy young men from rural areas who are generally not good at words. In order to praise customers, they have to overcome their inner aversion and show their own positive emotions to face customers. Such compromise itself is a kind of invisible emotional labor. In addition, takeaway delivery staff have repressed run may be in the process of single platform, merchants or the third party on the negative emotions, such as meals, such as anxiety, the businessman sent the wrong one by mistake injustice, system is not reasonable to send single cause of be agitated, and even see peer distribution had an accident in the process of the fear and worry, bad weather and traffic conditions meals difficult and not well, these complex emotions in a short period of time, no one can talk to palliate also did not have a good working environment, they can only digest alone in the process of delivery, in order to avoid a bad consumption experience for the customer.

\section{CURRENT SITUATION AND OPTIMIZATION PATH OF EMOTIONAL LABOR MANAGEMENT}

In order to survive and develop in the fierce takeaway industry, the takeaway platform-based enterprises, as managers, put forward clear and detailed requirements for the daily working emotional standards of deliverers. "Five-star praise rate" is the most important means for the platform to manage the delivery personnel. For example, the Meituan takeout platform accumulates the score of the customer praise rate of the external salesman, and directly links the monthly points to the salary and bonus of the deliveryman. The basic score for a gift is 10 points, and an extra 20 points can be added for a five-star review. The website system of each platform will judge the "five-star general" every month according to the five-star praise rate of the delivery staff, and reward 200 yuan as a bonus. At the same time, the "bad review" mechanism is also very strict. 50 points will be deducted for a bad rating and fines ranging from 50 to 500 yuan will be deducted based on customer complaints. This can be seen as the incentive and disciplinary management of the emotional labor of the customer in the commercial rules by the deliverer. The more sufficient the emotional labor, the 
more the reward. However, the platform does not provide corresponding management measures for the different emotional labor produced by the deliverer under other rules.

Emotional labor not only endangers the physical and mental health of delivery personnel and thus affects their work efficiency[7], but also may affect the effective operation of the delivery platform. What's more, the uncontrolled emotional labor of delivery personnel may even threaten the lives and property safety of businesses and customers. In view of this, this paper believes that the management of emotional labor can be optimized from the organizational level and employee level.

\subsection{Optimization of Emotional Labor Management at the Organizational Level}

The root of the emotional stress of the delivery staff is the delivery time and route navigation set by the intelligent delivery system. Platform should be to keep up with the idea of humanistic management widely listen to the use of takeaway delivery staff, optimization scheduling system, under the bad weather to delivery staff, set aside time elasticity and provide incentives for some outstanding distribution member and individual orders overtime exemption, and upgrade delivery staff appeal function, timeout, complaints of special circumstances to verify again, make its member assessment does not affect the distribution and income. On the route navigation, we do not blindly pursue time and speed, but improve the route navigation to make it fit the reality, more scientific and reasonable, so as to relieve pressure for the delivery staff.

Studies have confirmed that psychological capital (confidence, hope, optimism, resilience) has a significant inhibitory effect on negative behaviors similar to job burnout (emotional exhaustion, cynicism, and low sense of achievement). It is necessary to select employees with high psychological capital level. During the recruitment process, the platform should recruit employees with full psychological capital, or help them to build their psychological capital through professional training, and make psychological correction and psychological counseling for them regularly, so as to keep their emotional state in a full position. The platform should also inject "energy" into the deliverers' "emotional labor pool". Food delivery personnel's efforts often result in the loss of physical and mental resources, and the return can realize the effective supplement of resources. Delivery platform can be delivered by improving the member benefits, paid vacation, provide training and development opportunities, make food distribution cost as a result of long-term emotional labor resources get timely and effective supplement of body and mind, reduce the emotional labor have negative consequences for employees, can delivery platform set up the good enterprise image in the society and the social sense of responsibility.

\subsection{Optimization of Emotional Labor Management at the Employee Level}

For the deliverer, in the face of customers, merchants, platforms and other third-party personnel, the inconsistency between the false emotional expression and the inner reality brought by wearing the mask will consume more psychological resources and aggravate the psychological fatigue[8]. By actively adjusting their own subjective experience and inner expression, and providing services from the heart, to achieve an active deep performance that is consistent with the outside, the takeaway deliverers can improve their personal sense of accomplishment and job satisfaction[9]. Delivery personnel should strengthen their own active emotional labor strategy, and perform more deep roles in the process of delivery, so as to meet the requirements under various rules. Active emotional labor strategy can also arouse customers' positive emotions, give more praise to the delivery staff, and play a positive incentive role.

\section{CONCLUSIONS}

This paper first introduces the current situation of takeaway deliverers. Then, from the perspective of emotional labor, it studies the formation of emotional labor of deliverers who sell to others from the perspectives of organization, business and society, and discusses the optimization of emotional labor management from the perspectives of organization and employees. The research finds that under the organizational rules, the time standard and distribution rules formulated by the platform accelerate the formation of emotional labor of the deliverer. Under the commercial rules, customers' favorable or non-negative comments are the main reasons for their emotional labor, and the takeout vendors will also add content to their emotional labor. The emotional labor under social rules is in a dynamic change, and the deliverer needs to digest the invisible emotional labor alone. At present, the emotional labor management mechanism of the organization (takeaway platform) for employees (takeaway deliverers) has not been improved, and time constraint is greater than everything. In order to optimize the management, the organization should comply with the humanistic management idea, improve the algorithm, relieve pressure for employees, pay attention to the construction of employees' psychological capital, and inject "energy" into the delivery staff by improving employee welfare, paid vacation and other ways. Employees should adopt more active emotional labor strategies to provide services for customers from the bottom of their hearts. 


\section{AUTHORS' CONTRIBUTIONS}

Previous studies did not discuss the content and formation of the emotional labor of delivery workers, nor did it discuss the emotional labor management of the delivery industry. This article has carried out a research on this gap in the literature, focusing on the staff in the delivery and delivery field of the service industry. Management research has played a perfect and complementary role.

\section{ACKNOWLEDGMENTS}

This work was supported by the [Major Program of The National Social Science Fund of China \#1] under Grant [No. 15ZDA022].

\section{REFERENCES}

[1] J.H. Shen, Young migrant workers on wheels: A study on the labor process of takeaway riders. Youth Development Forum, 2019, (05): 69-76.

[2] Hochschild, A. The Sociology of Feeling and Emotion: Selected Possibilities, New York: Anchor, 1975, 280- 307

[3] X.Y. Li, Research on the relationship between psychological capital, emotional labor strategies and job burnout. Management Science, 2013.

[4] H.Y. Wu, "Double-sided" management of emotional labor. Enterprise Management, 2009 (09): 28-29

[5] P. Sun, Digital labor under "algorithm logic": A study of food delivery staff under the platform economy. Ideological front

[6] R. Zhang, Emotional Labor Management: A New Perspective of Human Resource Management in Non-profit Organizations. Journal of Zhejiang University, 2012.

[7] L.X. Wang, Discussion on the Connotation of Emotion Management. Social Psychological Science, 2010.

[8] F.R. Liu, The theoretical source of Affective labor and the progress of foreign research. News, 2019, (12): $72-84$.

[9] Adams, I.T. and Mastracci, S.H. (2020), "Contrasting emotional labor and burnout in civilian and sworn law enforcement personnel",
Policing: An International Journal, Vol. 43 No. 2, pp. 314-329. https://doi.org/10.1108/PIJPSM-062019-0094

[10] Lee, L. and Madera, J.M. (2019), "Faking it or feeling it: The emotional displays of surface and deep acting on stress and engagement", International Journal of Contemporary Hospitality Management, Vol. 31 No. 4, pp. 1744-1762. https://doi.org/10.1108/IJCHM-05-2018-0405

[11] Hayes, S. and Kleiner, B.H. (2001), "The managed heart: the commercialisation of human feeling and its dangers", Management Research News, Vol. 24 No. 3/4, pp. 81-85. https://doi.org/10.1108/01409170110782667

[12] Kinman, G. (2009), "Emotional labor and strain in "front-line" service employees: Does mode of delivery matter?" Journal of Managerial Psychology, Vol. 24 No. 2, pp. 118-135. https://doi.org/10.1108/02683940910928847

[13] Seery, B.L. and Corrigall, E.A. (2009), "Emotional labor: links to work attitudes and emotional exhaustion", Journal of Managerial Psychology, Vol. 24 No. 8, pp. 797-813. https://doi.org/10.1108/02683940910996806

[14] Hochschild, A. Explore The Managed Heart, Berkeley: University of California Press. 1983, 305- 314

[15] Hur, W.-M., Moon, T.-W. and Jung, Y.S. (2015), "Customer response to employee emotional labor: the structural relationship between emotional labor, job satisfaction, and customer satisfaction", Journal of Services Marketing, Vol. 29 No. 1, pp. 71-80. https://doi.org/10.1108/JSM-07-2013-0161

[16] Kumar Mishra, S. (2014), "Linking perceived organizational support to emotional labor", Personnel Review, Vol. 43 No. 6, pp. 845-860. https://doi.org/10.1108/PR-09-2012-0160

[17] Moon, T.W., Hur, W.-M. and Choi, Y.J. (2019), "How leaders' perceived emotional labor leads to followers' job performance: A serial mediation model", Journal of Service Theory and Practice, Vol. 29 No. $1, \quad$ pp. 22-44. https://doi.org/10.1108/JSTP-11-2017-0201 